\title{
ION SOURCE FOR A SINGLE PARTICLE ACCELERATOR
}

\author{
TOMÁŠ MATLOCHA ${ }^{a, b}$ \\ ${ }^{a}$ Faculty of Nuclear Sciences and Physical Engineering, Czech Technical University in Prague, Břehová 7, \\ 11519 Praha 1, Czech Republic \\ ${ }^{b}$ Nuclear Physics Institute of the CAS, 25068 Rež u Prahy, Czech Republic \\ correspondence: matlocha@ujf.cas.cz
}

\begin{abstract}
This paper proposes a method to obtain very low beam current on the injection side of an particle accelerator. By using a combination of a well known ion source type and a short photo-emissive laser pulse, very low amount of ions can be created. The method could be used in the ion source of various types of the accelerators, where very low beam current is essential. The design is adapted to build a compact internal ion source and the dimensions of the device are adjusted to fit central region of the cyclotron U-120M. The functional parameters of the device are discussed and the amount of the produced ions is estimated.
\end{abstract}

KEYWORDS: single particle accelerator, cyclotron ion source, low beam current, laser ion source, pulsed ion source, U-120M, proton radiography.

\section{INTRODUCTION}

This paper presents a principle of delivering a very low beam current to the injection system of a single stage or low energy accelerator. A control of the ion creation process is a complex task with effect of many parameters. One of the control parameter can be the length of the primary ionizing pulse in the ion source. This length is naturally restricted and usualy can not be arbitrary lowered. When the ionizing pulse is driven using a high voltage discharge, the pulse length with duration about tens of nanosecond can hardly be achieved. From this reason, a primary ionizing plasma in presented ion source is not made using ordinary high voltage discharge, but using a laser pulse. With the present-day laser systems, the high intensity laser pulses with the duration of few ps could be generated and used for photo-emission. Electrons from photo-emission then controllably ionize gas and produce ions for the low energy proton or heavier particle accelerator.

A usage of the low current beams vary accordingly with the technical field where the beam is applied. The low current beams are required in the radio-biological microbeam study of the living cells [1, in the single proton detectors tests or in the proton radiography. A benefit of using proton beams instead of classical gamma-rays in the radiography, is that the scanned sample receive significantly lower dose for similar image result [2]. With a proper magneto-optic lens system, very high-resolution images could be obtained. Usual resolution even with the low energy proton beams is several tens of $\mu \mathrm{m}$. This resolution of the proton radiography method could be apparently further improved and its limits are far beyond present ranges [3. Very good resolution of the proton radiography [4] could lead to a specific high resolution 3D imaging, where a complete 3D image of the sample could be obtained by two or three laser shots in the proposed ion source. For a very small and sensitive scanned samples, the lowest possible irradiation can be important, so precise control of the number of the created ions is essential. Schematic principle of the proton radiography is shown in Fig. 1.

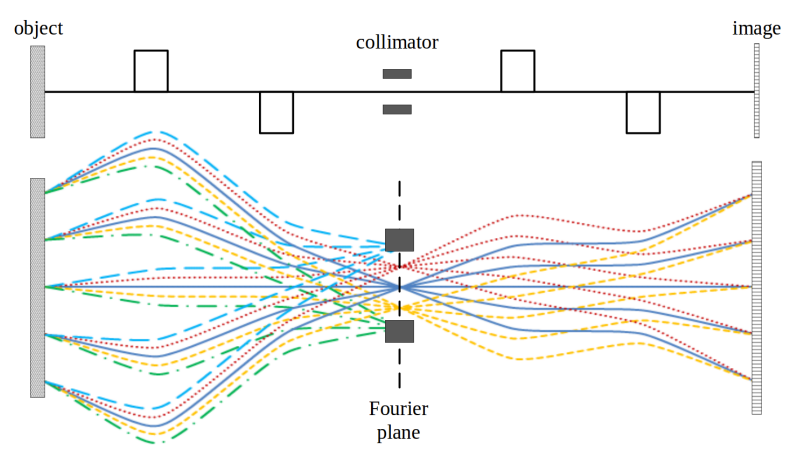

Figure 1. The proton beam lens system with the particle trajectories as colored lines [3].

\section{Beam Current Limitation}

The idea presented in this paper has grown from the response tests of the silicone detectors and electronics for the planed upgrade of the Inner Tracking System of the ALICE detector [5]. The tests were performed on the old cyclotron U-120M, shown in Fig. 2 These tests however require very low intensity of the delivered beam current [6]. A minimal beam current which can be delivered comfortably by this cyclotron is ca. $2.5 \mathrm{fA} / \mathrm{cm}^{2}$, which corresponds to ca. 15600 protons per second per $\mathrm{cm}^{2}$. The beam intensity is usually decreased only after its extraction from the ion source. It means that regardless of the output cyclotron beam current, the amount of ions at the 


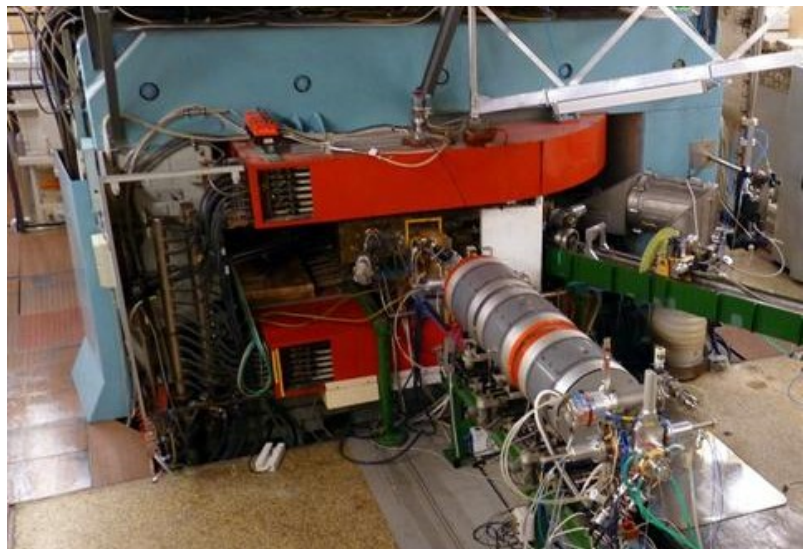

Figure 2. Cyclotron U-120M.

beginning of the acceleration process is almost the same. This factor limits stability of the accelerator and reduces utilization of low current beams. The Laser Induced Photo-emission Ion Source presented in this paper, can significantly reduce the number of ions entering the machine.

\subsection{U-120M PIG ION SOURCE}

The ion source of the cyclotron is a movable part and its position can be fine-adjusted with respect to the puller. The extraction window of the ion source has area approx. $2 \mathrm{~mm}^{2}$. The puller has an input window with an area of about $20 \mathrm{~mm}^{2}$. An optimal position of the ion source opposite to the puller guarantees a maximal extraction to loss ratio. In normal operation this extraction ratio is kept as high as possible. When the low current beam is required, this ratio is lowered. This is achieved mainly by changing the position of the ion source extraction window. The ion source is moved slightly to one side, so the extraction window of the ion source does not overlap with the puller input window. Subsequently, only a minimal number of ions are able to pass the initial extraction. The rest of the ions are lost on the puller body as can be seen in Fig. 3 and 4

Although the above mentioned current regulation is very easy, it has some disadvantages. The U-120M cyclotron ion source is a Penning Ion Gauge (PIG) type ion source with a cold cathode, operating in continuous regime. In Fig. 5 is photo of the ion source oriented with its head on the left side, and in Fig. 6 is its head detail.

The ion source has a cylindrical plasma chamber parallel to the cyclotron magnetic field of approx. 1 T. The chamber is enclosed from both sides by tantalum cathodes. The cylindrical wall of the ion source is an anode and whole body is made from a tungsten-copper composite. Electrons emitted from the cathodes are accelerated by the anode potential. Due the strong magnetic field the electrons are trapped by the field lines and are not able to directly reach the anode. The electrons repeatedly oscillates between the cathodes and are slowly drifting to the anode

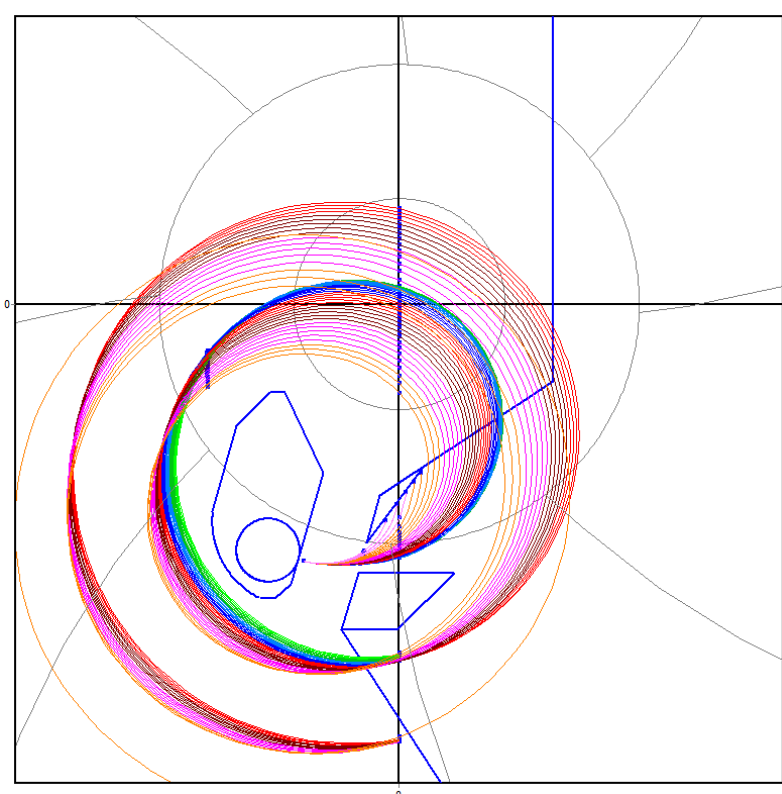

Figure 3. Central region top view - optimal extraxion for proton regime $37 \mathrm{MeV}$ simulated with Durycnm4 [7].

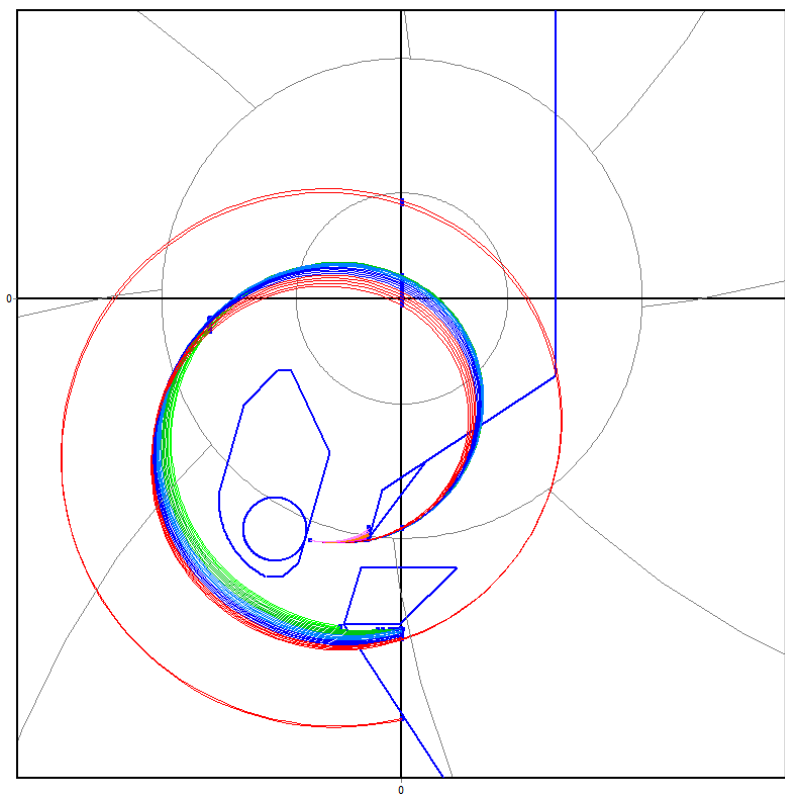

Figure 4. Central region top view - minimal extraxion for proton regime $37 \mathrm{MeV}$ simulated with Durycnm4 [7].

body. The electrons ionize the gas in the cylinder and create the plasma. The amount of the produced ions can be slightly regulated by changing the plasma current and by regulating the ion source gas presure. For stable operation, the Penning ion source needs some minimal plasma current to heat up cathodes and some minimal corresponding gas concentration. In the usual working regime the plasma current is about $1 \mathrm{~A}$ and the minimal plasma current for stable operation is several miliamperes.

Basically in the ion source are two concurrently 


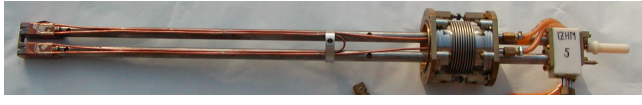

FigURE 5. U-120M PIG ion source.

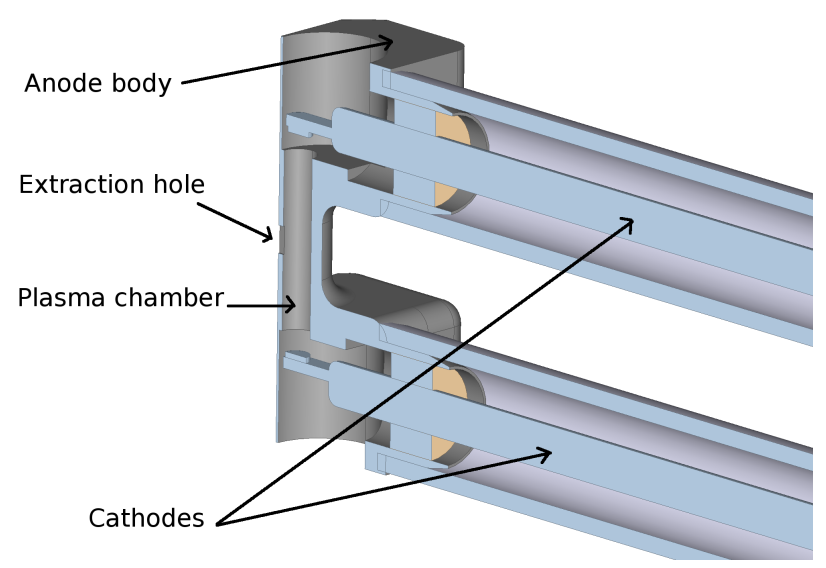

Figure 6. Current U-120M PIG ion source.

processes which lead to creation of ions. The first process is based on stripping orbital electrons of neutral atoms and generates positive ions. The degree of ionization is done by temperature of the plasma electrons and by a time of interaction. Our Penning ion source has plasma electron temperature up to approx. $70 \mathrm{eV}$. It is enough to produce ions with low ionization potentials, e.g. $\mathrm{H}^{+}, \mathrm{D}^{+}, \mathrm{He}^{2+}, \mathrm{Li}^{2+}, \mathrm{C}^{4+}$, etc. In second process, the negative ions are produced. The second process leads to production of negative ions via the Surface and the Volume production process. The Section 5.1 describes the negative hydrogen ions formation process in more detail.

After their creation, both negative and positive ions are simultaneously extracted from the ion source by an alternating RF high voltage field. Negative polarity extracts positive ions and oppositely. Polarity of the cyclotron magnetic field determines the type of ions which will be accelerated. The second type of ions are simply extinguished on the Puller body.

\subsection{Pulsed PIG ION SOURCE}

From above mentioned Penning ion source operation principle it follows, that the ions are produced permanently. From that reason the lowest current of extracted ions is limited from below. There were several attempts to switch our Penning ion source to a pulsed operation mode. These studies were motivated by other reasons than lowering the output current, but a deeper knowledge of the PIG pulse regime capabilities were gained. An ignition process of the PIG ion source from the full Off state is very long and lasts couple of seconds. When the PIG plasma is once ignited, the plasma current can be lowered to five miliamperes, and the ion source is in low current state. In this state changing of the plasma current can be done quickly with a reaction time of a few microseconds. Then the ion source can be fast and easily modulated between low and high current states. But the ion source even in the low plasma current mode is still producing much more ions than required. There is an option to further lower the low current value by at least an order of magnitude by driving the cathodes temperature. Nevertheless, continuous operation of the ion source is not suitable for the low current beams.

From this reason, the possibilities of other ion production were studied and the main findings are presented in the next paragraphs.

\section{INSPIRING ION SOURCES}

\subsection{EBIS/EBIT ION SOURCE}

A principle of operation of an Electron Beam Ion Source (EBIS) and an Electron Beam Ion Trap (EBIT) is very similar. The electrons are extracted from a cathode by thermionic emission and accelerated in a strong solenoidal magnetic field by an anode voltage. The shape of the magnetic field forms the entering electrons to well focused beam with densities up to several $\mathrm{kA} / \mathrm{cm}^{2}$. This beam ionizes the gas and forms the ions. Because the electron beam temperature can be very high, a high ion charge state is possible. In the EBIT the ions are confined in a space charge of a dense electron beam, where attractive force of electrons prevent the ions to move. An electrostatic potential of external electrodes is applied to trap the ions and hold their position. When the trapping potential is switched off, the ions can be extracted. This type of ion source is mainly used for heavy ions for which high charge states or full stripping by other techniques is complicated. Temperature of electrons could be from $10 \mathrm{keV}$ to $200 \mathrm{keV}$. The extracted ion current is low, typically ranging from $10^{5}$ to $10^{9}$ ions per second. The EBIS/EBIT sources exist in many designs from ultra compact devices for low current and low charge states to huge machines capable of delivering fully stripped uranium ions [8, 9]. Principial schematic of the EBIT is in Fig. 7

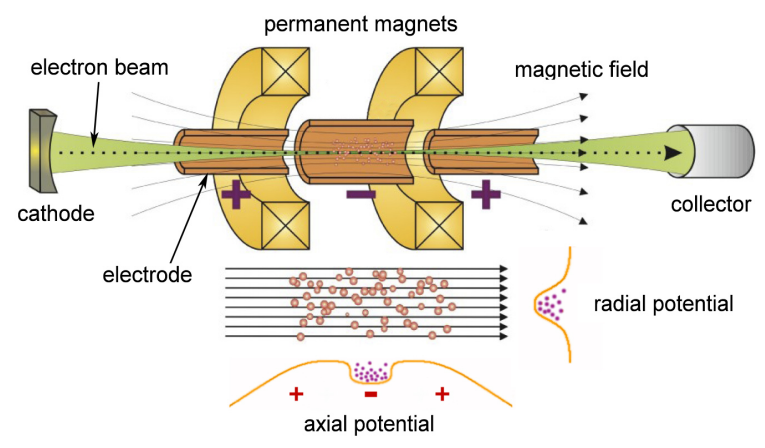

Figure 7. Schematic design of EBIT ion source [10]. 


\subsection{RF GUN}

Modern light sources such as the Energy Recovery Linac (ERL) or Free Electron Lasers (FEL) use as an electron beam generator pulse operated RF Guns. The RF Gun is a laser induced photo emission electron gun, where photo electrons are accelerated by a strong electric field generated during the RF pulse. Schematic design is shown in Fig. 8 The photosensitive cathode is placed near the center of a $3 / 2$ cell RF cavity $(0.5-2 \mathrm{GHz})$, where a high electric field is produced (up to $120 \mathrm{MV} / \mathrm{m}$ ). A very short laser pulse $(10 \mathrm{ps})$ with a proper phase is applied onto the photo-cathode. A bunch of photoelectrons is emitted, accelerated to proper energy and delivered further into the system. Currently exist a number of devices of this type, capable to produce electron beams with energies in the range $0.1-5 \mathrm{MeV}$.

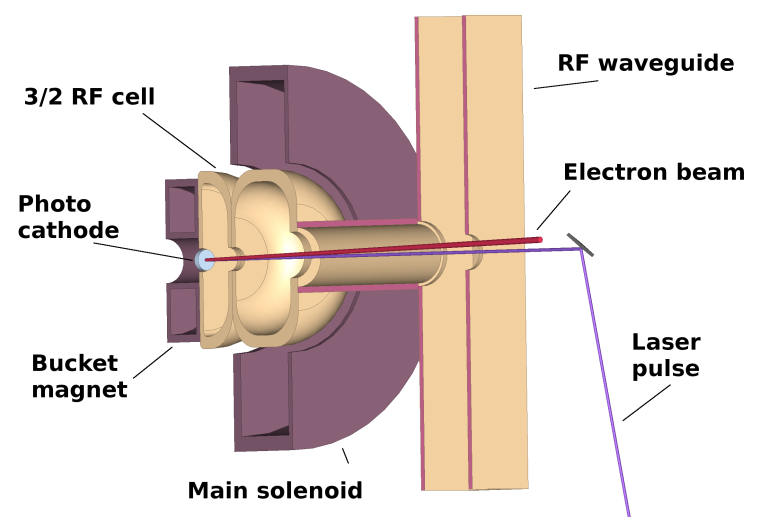

Figure 8. Schematic design of the RF gun.

Photo-cathodes used in these electron sources can be from metal or semiconductor. In general, metals have lower electron conversion efficiency(QE) and need UV lasers. On the other hand they have fast response and are resistant to impurities. Semiconductor cathodes are more efficient and can be operated with visible/IR laser, but have slower time response and are sensitive to contamination and photon radiation. Charge emitted per bunch is approx. $1 \mathrm{nC}$. Required peak RF power is up to $10 \mathrm{MW}$. Mean laser power is $1 \mathrm{~W}$ and required frequency is few thousands pulses per second [11, 12].

\section{LASER Induced Photo-Emission ION SOURCE}

The above mentioned devices could probably be combined. By applying a short laser pulse on a photo sensitive cathode one could produce an electron bunch. This bunch could be then accelerated to a desired energy by an anode potential. During travel from a photo-cathode to an anode, this bunch could ionize gas present in the discharge chamber. Because the electron temperature is expected only few tens $\mathrm{eV}$, sufficient to ionize hydrogen and helium, and emitted charge is low, the device could be kept compact. Such a compact device could be placed inside the central region of the cyclotron and in demand of very low beam currents replace the present PIG ion source. Dimensions of the PIG ion source must be preserved to fit into the cyclotron central region. These dimensions determine the discharge chamber shape and possible arrangement of the electrodes. Unlike in the PIG ion source, where the cylindrical chamber is anode enclosed by cathodes from both sides as in Fig. 6. here will be photo-cathode on one side and anode on the other side of the cylinder as in Fig. 9. In this configuration the electron bunch will pass through the chamber only once per one laser pulse. The laser pulses with energies of few $\mu \mathrm{J}$ are driven to the photo-cathode through ion source arms enclosed by a high vacuum sealed window, from an external laser generator placed out of the cyclotron site.

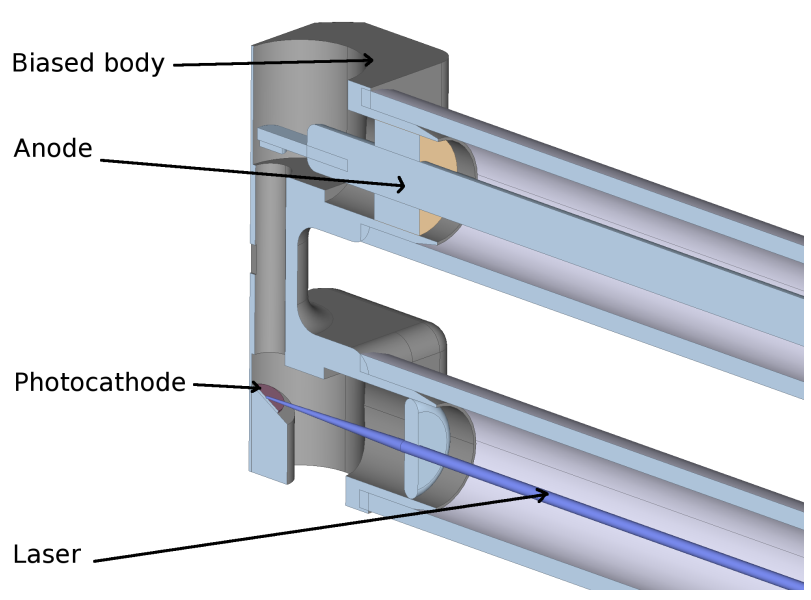

Figure 9. Proposed laser ion source.

\section{1. Рhoto-CAThode}

In general, the photo-cathode should have work function below $3 \mathrm{eV}$ to avoid a necessity of using UV lasers. Considering a gamma radiation always present at the cyclotron site, the photo-cathode should be metallic. Other reason for this choice is good resistance to adverse conditions such as bad vacuum and impurities in the system, where semi-conductors cathodes are very sensitive. In theory, the metal photo-cathodes should not work when irradiated by photons with energies below the work function $\mathrm{h} \nu<\Phi$. In reality, an effect of multiple photon absorption were observed in high density laser pulses, and even photons energies below the threshold energy can be used for photoemission [13]. The Quantum Efficiency is defined by ratio between incident photons and emitted electrons. The metals have QE low, but in our case it should be helpful for limiting a parasitic emission by photons captured from de-excitation and neutralization of the gas atoms. Copper, which has QE $5 \times 10^{-5}$ at wavelength $320 \mathrm{~nm}$, could be suitable for the first try. 


\section{IONS PRODUCTION}

\subsection{Negative hydrogen ION FORMation}

As the principle of proposed pulsed ion source is simple, many practical limitations will probably occur. The main questions are, if this device will be capable to produce any negative ions and how long this ions will live for.

A creation of the negative hydrogen ion $\mathrm{H}^{-}$is a multi-step process, where several conditions should be fulfilled. There are many processes involved in $\mathrm{H}^{-}$formation. One of them, the Volume negative ion production process which needs at least two different temperature of electrons. Faster electron with energies near $20 \mathrm{eV}$ which ro-vibrationally excites the hydrogen $\mathrm{H}_{2}^{*}$ and a cold electron, which can than dissociate the $\mathrm{H}_{2}$ molecule and attach to one of the atoms to form an negative hydrogen ion:

$$
\mathrm{H}_{2}+\mathrm{e}^{-} \longrightarrow \mathrm{H}_{2}^{*}+\mathrm{e}^{-} \longrightarrow \mathrm{H}^{0}+\mathrm{H}^{-} .
$$

This process is called a dissociative attachment process and the dissociative low temperature electron energy needs to be close to $1 \mathrm{eV}$. Bounding energy of the second electron in the $\mathrm{H}^{-}$ion is $0.75 \mathrm{eV}$, so the once formed $\mathrm{H}^{-}$ion can be easily destroyed by collisions with other ion or on residual gas.

The second important $\mathrm{H}^{-}$formation process is the Surface negative ion production. In this process the previously formed $\mathrm{H}^{0}$ atom from $\mathrm{H}_{2}$ dissociated molecule collides with a metallic surface of the plasma chamber. When bouncing back from the wall, an conductive electron from the metal surface can be trapped by the atom and form an $\mathrm{H}^{-}$ion. Work function of the chamber metal tungsten surface is near $4.5 \mathrm{eV}$, far above $0.75 \mathrm{eV}$ electron affinity of $\mathrm{H}$ atom. From this reason the cross-section of $\mathrm{H}^{-}$formation from pure metal surfaces is commonly increased by covering surface with caesium layer. This thin Cs layer lowers the work function of surface to approx. $1.6 \mathrm{eV}$. The Cs is widely used in $\mathrm{H}^{-}$ion sources and has its significant role also in the Volume $\mathrm{H}^{-}$production. In both processes significantly increases $\mathrm{H}^{-}$formation probability. In the U-120M cyclotron Penning ion source Cs is not used, so the Volume process is therefore considered to be more important [14 16].

\subsection{Positive iOns FORMATION}

A situation with positive ions should be more simple. Ionization of a low atomic number elements is done by stripping their orbital electrons in energetic collisions. As for heavier elements the process is gradual, because the atoms are ionized in a stepwise processes where each electron collision removes one or two orbital electrons. For light atoms such as $\mathrm{H}$ and $\mathrm{He}$, this can be done very fast. Ionization energy for hydrogen is $13.6 \mathrm{eV}$ and has maximal cross-section $\sigma=0.6 \times 10^{-16} \mathrm{~cm}^{2}$ at $50 \mathrm{eV}$. Full ionization energy for helium is $54.4 \mathrm{eV}$ and its maximal cross-section $\sigma=0.3 \times 10^{-16} \mathrm{~cm}^{2}$ is near $100 \mathrm{eV}$ [17.

\subsection{IONS YIELD ESTIMATION}

For the laser pulses with duration $20 \mathrm{ps}$ the emitted charge $100 \mathrm{pC}$ could be expected. With optimal electron temperature and for $\mathrm{H}_{2}$ concentration in range from $10^{9} \mathrm{~cm}^{-3}$ to $10^{13} \mathrm{~cm}^{-3}$ [18] in a volume similar to current used PIG ion source $2 \mathrm{~cm}^{3}$, from current density $18 \mathrm{~A} / \mathrm{cm}^{2}$ there can expected from $10^{2}$ to $10^{5}$ positive ions. In normal operation the ratio between protons and $\mathrm{H}^{-}$ions is $10^{-2}$. Very low probability of formation $\mathrm{H}^{-}$ion in the single pulse can be expected. Even if the production ratio is lowered by two orders to $10^{-4}$, still up to $10 \mathrm{H}^{-}$ions per volume could be expected. With considering a fact, than an exact $\mathrm{H}^{-}$ formation process understanding is still in progress, an influence of more factors or processes, such as a wall biasing, could play its role. From this reason a formation of an $\mathrm{H}^{-}$ion in the single discharge bunch should not be rejected in advance.

\subsection{Discharge CONDITIONS AND IONS EXTRACTION}

After formation of the electron bunch by an irradiation of the photo-cathode, a multiple side effects can be expected. One from these effects could be the above mentioned parasitic photo-emission. Other important effect will be positive ion bombardment of the cathode. Parameter which can highly affect performance of the device will be a lifetime of produced ions which will probably reduce a minimal length of the ion pulse.

\section{Beam Time Distribution}

The low current regime of $\mathrm{H}^{-}$ions with energy $36.9 \mathrm{MeV}$ has its cyclotron frequency $25.8 \mathrm{MHz}$. This main carrier frequency is modulated by a $150 \mathrm{~Hz}$ signal. Particles in the cyclotron are accelerated with each half period and are caught in phase angle of about from $-30^{\circ}$ to $30^{\circ}$ of this half period. The ions are revolving in approx. 6 ns micro-bunches with $32 \mathrm{~ns}$ spaces between them. With $10 \%$ carrier modulation these micro-bunches are formed into $0.67 \mathrm{~ms}$ long bunches with $6 \mathrm{~ms}$ spaces.

A simulation of the cyclotron response to a $110 \mathrm{ps}$ short input pulse with 330 ions was made in the code Durycnm4 [7, 19]. The ions from one input pulse are accelerated for 503-507 periods and divided into five output micro-bunches. Output signal than lasts for $160 \mathrm{~ns}$. Energy range of the particles is from 36.75 to $36.9 \mathrm{MeV}$.

This cyclotron impulse response is made by a phase instabilities and space distribution of the micro-bunch, which affects energy increment difference particle gains each turn and leads to energy spread of the beam. Because the extraction is made on the desired energy, each particle from the input bunch needs a different number of the accelerating turns. As a result on the output, the signal is time dispersed. This dispersion is coming from the machine nature and cannot be avoided by shortening of the input pulse. Number of ions in individual micro-bunches are shown in Fig. 10. 


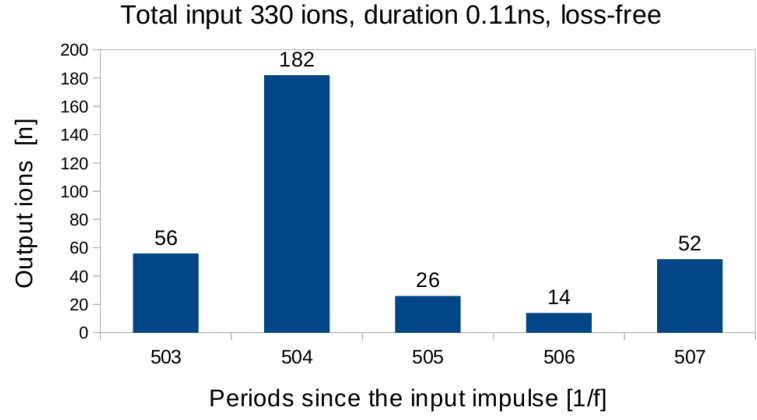

FIGURE 10. Simulated U-120M impulse response.

\section{Conclusions}

This paper proposes a method which possibly could be used to produce a very small amount of the ions. Considering many variable parameters that can be used to tune the ion source, it can be expected that the ions could be produced in countable amounts and delivered to the accelerating process on the request. The benefits of the presented method is mainly in the controlled production of the selected charge state and the number of the generated ions. As the demand of low current ion beams is increasing rapidly, overcoming the difficulties with generation the ions by the laser induced photo-emission could be worth the effort.

\section{REFERENCES}

[1] G. A. Drexler, C. Siebenwirth, S. E. Drexler, et al. Live cell imaging at the munich ion microbeam SNAKE - a status report. Radiation Oncology 10(1):42, 2015. DOI:10.1186/s13014-015-0350-7

[2] N. Depauw, J. Seco. Sensitivity study of proton radiography and comparison with $\mathrm{kV}$ and $\mathrm{MV}$ x-ray imaging using GEANT4 monte carlo simulations. Physics in Medicine and Biology 56(8):2407, 2011. DOI:10.1088/0031-9155/56/8/006

[3] T. Wei, G.-J. Yang, Y.-D. Li, et al. First experimental research in low energy proton radiography. Chinese Physics C 38(8):087003, 2014. DOI:10.1088/1674-1137/38/8/087003.

[4] N. Depauw, V. Taranenko, S. Danto, et al. TH-D-201B-03: A comparison of protons versus gamma $\mathrm{x}$-rays in producing patient radiographs: A monte carlo study. Medical Physics 37(6):3472-3472, 2010. DOI:10.1118/1.3469562

[5] B. Abelev, et al. (The ALICE Collaboration). Technical design report for the upgrade of the ALICE inner tracking system. Journal of Physics G: Nuclear and Particle Physics 41(8):087002, 2014. DOI:10.1088/0954-3899/41/8/087002.

[6] T. Vanát, J. Pospíšil, F. Kř́žzek, et al. A system for radiation testing and physical fault injection into the FPGAs and other electronics. In 2015 Euromicro
Conference on Digital System Design (DSD), pp. 205-210. 2015. DOI:10.1109/DSD.2015.98.

[7] Nuclear Physics Institute of the CAS. Mathematical simulation and analysis of the cyclotron U-120M, 2010. http://accs.ujf.cas.cz/index.php/en/component/ content/article/8.

[8] The National Institute of Standards and Technology. NIST EBIT introduction. http://www.nist.gov/pml/div684/grp01/intro.cfm

[9] Lawrence Livermore National Laboratory. Electron beam ion trap (EBIT). LLNL-WEB-409815 Rev. 1, https://www-pls.llnl.gov/?url=about_ pls-physics_division-hed_physics-ebit.

[10] Highly charged Ion Trapping facility, GSI. The SPARC EBIT. http://web-docs.gsi.de/ stoe_exp/ laboratory/environment/ebit/ebit.php

[11] B. Dunham. Introduction to electron guns for accelerators. Cornell University lecture notes, http://www . lepp. cornell . edu/ hoff/LECTURES/08S_ 688/08S_688_080225.pdf

[12] J. Teichert, A. Arnold, J. Stephan, et al. Progress of the Rossendorf SRF gun project. In Proceedings of the 27th International Free Electron Laser Conference, pp. 534-537. 2005.

[13] F. Le Pimpec, C. J. Milne, C. P. Hauri, F. Ardana-Lamas. Quantum efficiency of technical metal photocathodes under laser irradiation of various wavelengths. Applied Physics A 112(3):647-661, 2013. DOI:10.1007/s00339-013-7600-z

[14] C. W. Schmidt. Review of negative hydrogen ion sources. In Proceedings of the Linear Accelerator Conference, Albuquerque, New Mexico, USA, pp. 259-263. 1990.

[15] M. P. Stockli. Volume and surface-enhanced volume negative ion sources. In Proceedings of CAS-CERN Accelerator School: Ion Sources, pp. 265-284. 2013. DOI:10.5170/CERN-2013-007.265.

[16] D. P. Moehs, J. Peters, J. Sherman. Negative hydrogen ion sources for accelerators. IEEE Transactions on Plasma Science 33(6):1786-1798, 2005. DOI:10.1109/TPS.2005.860067.

[17] Physical Measurement Laboratory, NIST. Electron-impact ionization cross sections. http://physics.nist.gov/cgi-bin/Ionization/ion_ data.php?id=HI\&ision=I\&initial=\&total=Y

[18] M. Bacal, A. Hatayama, J. Peters. Volume production negative hydrogen ion sources. IEEE Transactions on Plasma Science 33(6):1845-1871, 2005. DOI:10.1109/TPS.2005.860069.

[19] M. Čihák, O. Lebeda, J. Štursa. Beam dynamic simulation in the isochronous cyclotron $\mathrm{u}-120 \mathrm{~m}$. In Cyclotrons and Their Applications, Eighteenth International Conference, pp. 385-387. 2007. http://accelconf.web.cern.ch/AccelConf/c07/ PAPERS/385.pdf 